\title{
Five cases of paediatric cervical spine distraction injury
}

S Andronikou

Red Cross Children's Hospital

University of Cape Town

Department of Paediatric Radiology

\section{S Dix-Peek}

Red Cross Children's Hospital University of Cape Town

D Meerkotter Johannesburg Hospital University of the Witwatersrand Department of Radiology

\section{Abstract}

Cervical spine

distraction injury was considered rare in children, but current literature claims that atlanto-occipital distraction is a major contributor to paediatric trauma mortality. Lower cervical spine distraction is considered rare and usually results in quadriplegia. Three cases of atlantooccipital distraction are presented here, supporting these claims. The two cases of lower cervical spine distraction are presented as good examples of a rare injury that is sustained in the same manner as atlantooccipital distraction, but may result in survival of the patient with quadriplegia.

\section{Case report}

Case 1

A three-year-old female was involved in a motor vehicle accident as a passenger. She was removed from the vehicle without prior stabilisation of the cervical spine. She presented to the emergency unit with quadriparesis, but stable vital signs. Examination revealed absent motor and sensory activity in all four limbs. The lateral cervical spine film and chest X-ray demonstrated a widened interspace at the $\mathrm{C} 6 / 7$ level with posterior listhesis of $\mathrm{C} 6$ on $\mathrm{C} 7$. MRI showed complete transection of the cord at the C4 and C6/7 levels associated with disruption of the posterior longitudinal and interspinous ligaments. The child's condition deteriorated and mechanical ventilation was instituted. At the time of this report the patient remains quadriplegic.

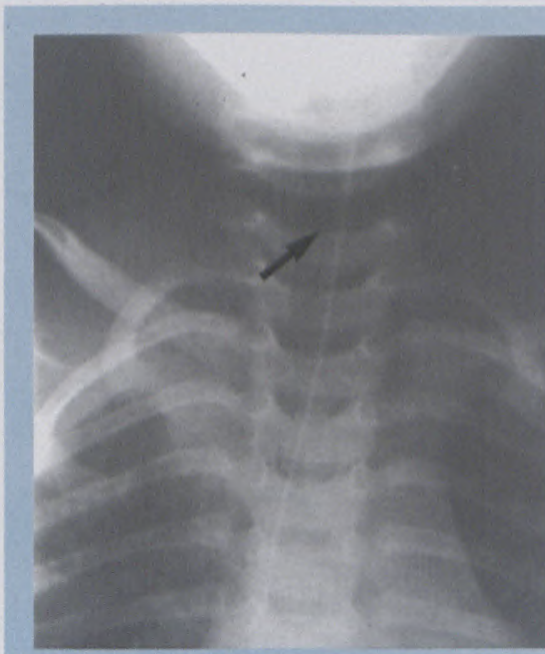

Figure 1: (Case 1) AP plain radiograph showing a widened interspace at C6/7 (arrow)

to page 9 


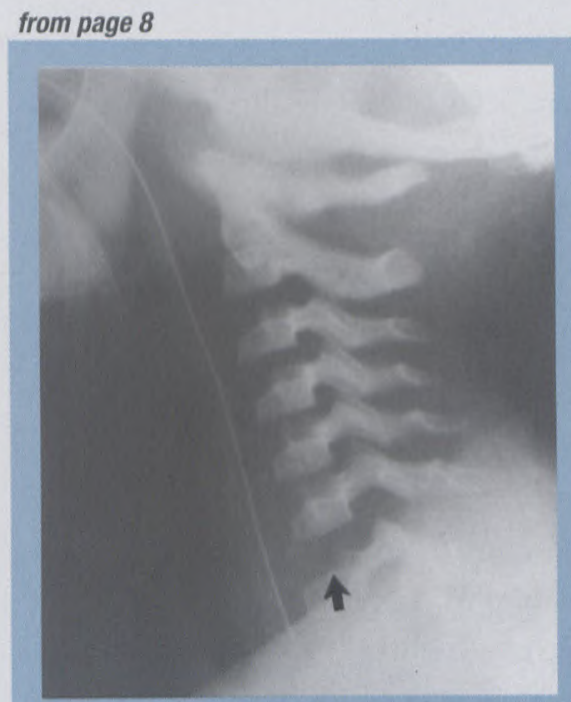

Figure 2: (Case 1) Lateral plain radiograph showing a wide interspace at $\mathrm{C} 6 / 7$ and subluxation of $\mathrm{C} 6$ on $\mathrm{C} 7$ (arrow)

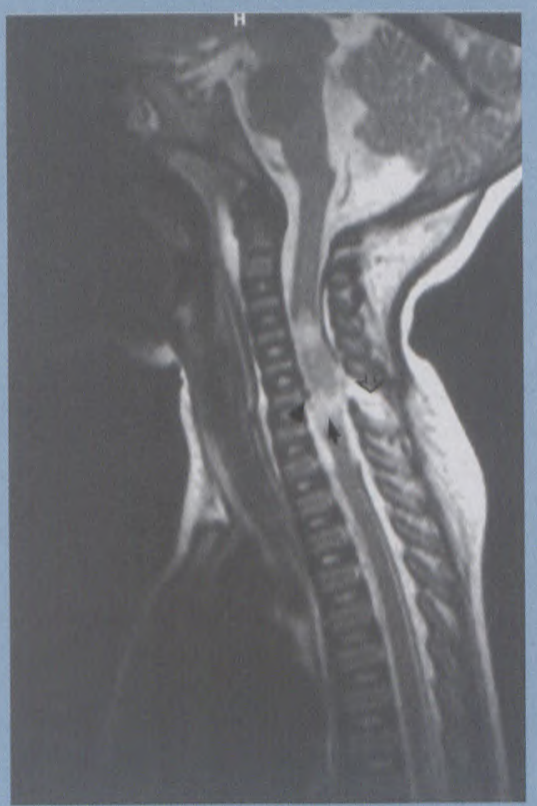

Figure 3: (Case 1) Saggital T2 weighted MRI showing complete cord transecton (closed arrow) and disruption of the posterior longitudinal (arrowhead) and interspinous ligament (open arrow)

\section{Case 2}

A three-year-old male was caught under a reversing vehicle. On examination the vital signs were stable but there was flaccid quadriparesis. Lateral cervical radiograph revealed distraction of the lower cervical spine at the $\mathrm{C} 6 / 7$ interspace. The patient died as a result of cardiorespiratory arrest 48 hours later.

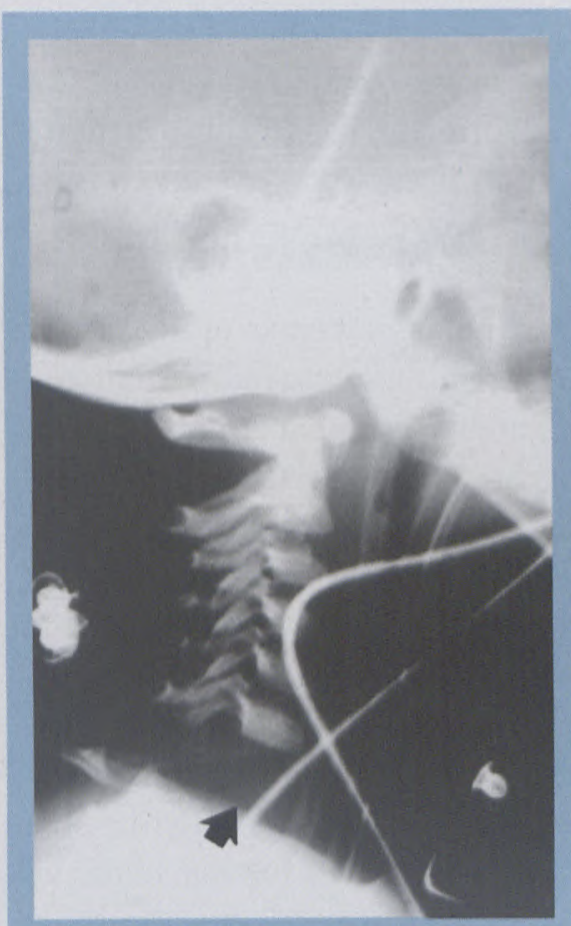

Figure 4: (Case 2) Plain radiograph demonstrates wide interspace at $\mathrm{C} 6 / 7$ (arrow)

\section{Case 3}

A five-year-and-ten-month-old female patient was involved in a motor vehicle accident as an unrestrained passenger. Bystanders attempted resuscitation and paramedics intubated the child and transferred her to the emergency department. On arrival the patient's pupils were fixed and dilated

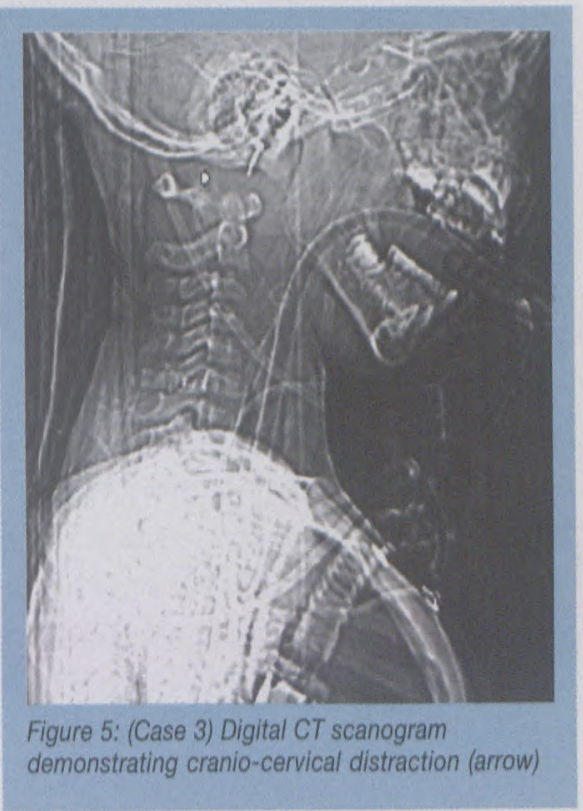

and the coma scale was calculated at its minimum (2T). A CT scan was performed to assess the intracranial compartment and a scanogram and CT of the cervical spine was obtained

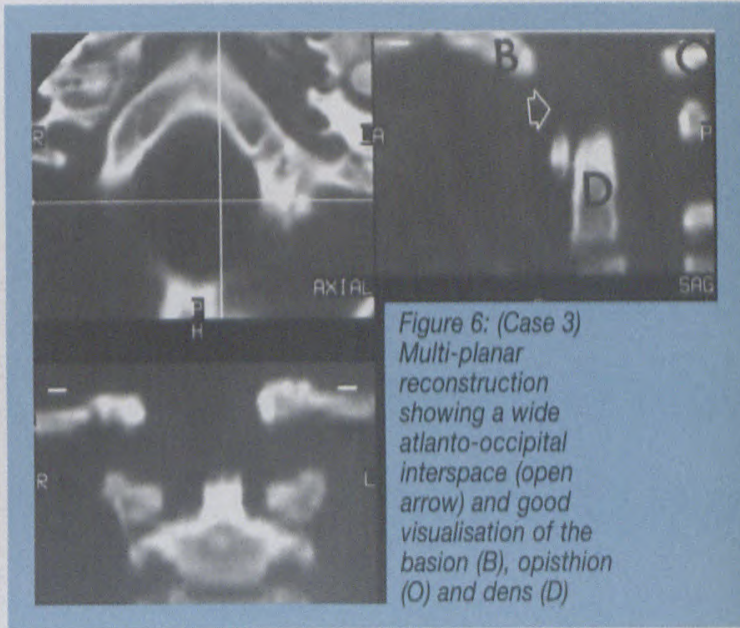

at the same examination. The scanogram demonstrated distraction at the cranio-cervical junction. Bradycardia ensued, but no further resuscitation was undertaken and the patient was declared dead at cardiac arrest.

\section{Case 4}

A nine-year-old female was involved in a motor vehicle accident as a pedestrian. She was intubated at the scene and was brought to the emergency department. Her blood pressure was low and temperature was decreased. Her coma scale was calculated at the minimum and her pupils were fixed and dilated. Her respiration was spontaneous. There were also numerous injuries to the appendicular skeleton. Neurosurgical assessment revealed no brainstem or spinal reflexes. The lateral cervical spine radiograph revealed massive prevertebral soft tissue swelling, and an increased interspace at the cranio-cervical articulation in keeping with a distraction injury. The patient then required assisted ventilation. The blood pressure dropped further the following 


\section{Five cases of paediatric cervical \\ spine distraction injury}

\section{from page 9}

day and when mechanical ventilation was terminated, the patient died.

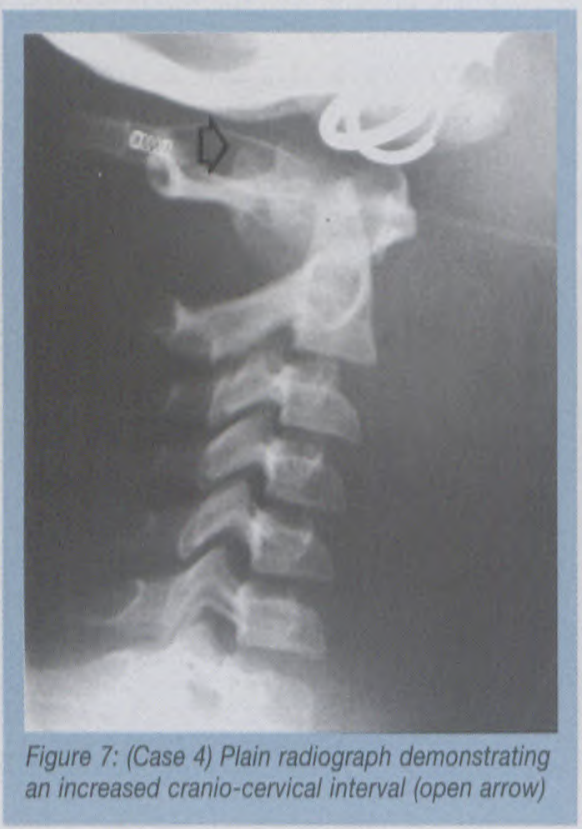

\section{Case 5}

This four-year-old female was involved in a motor vehicle accident as a pedestrian. She was intubated by ambulance personnel and brought to the accident unit with fixed dilated pupils and a minimum score on the Glasgow coma scale. Lateral cervical spine film showed distraction at the cranio-cervical junction Assisted ven-

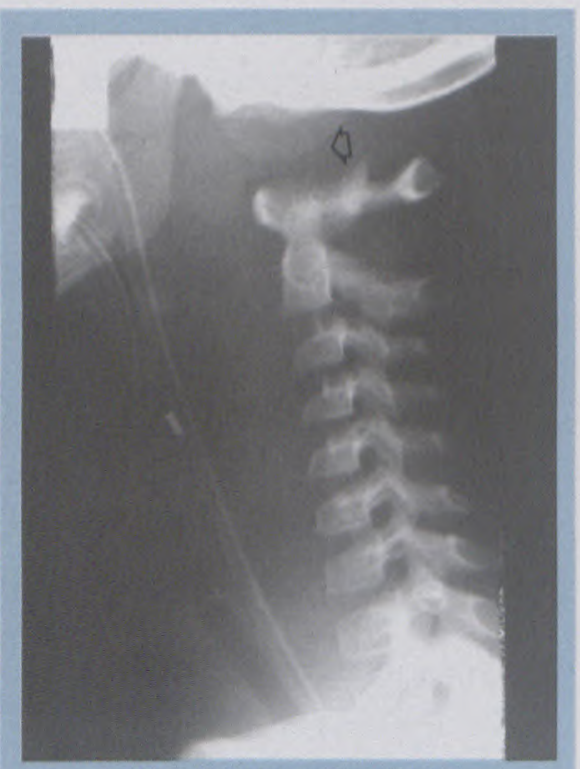

Figure 8: (Case 5) Plain lateral radiograph showing cranio-cervical distraction (open arrow) tilation was discontinued and no spontaneous respiration was noted. Cardiac arrest ensued and the patient was pronounced dead.

\section{Discussion}

The cervical spines of children differ from those of adults. Adult configuration is attained only at about eight years of age. ${ }^{1}$ Children are more prone to sustaining distractions, subluxation injuries and dislocations than adults. There are numerous factors accounting for this, including increased ligamentous laxity, horizontally oriented facets, hypoplastic occipital condyles and large head to body ratio. ${ }^{1,2,3}$ The fulcrum of motion in a child's cervical spine is around $\mathrm{C} 2 / 3$ and $\mathrm{C} 3 / 4$, whereas that of adults is lower. Injury to the Atlas and Axis is reported to represent $70 \%$ of cervical spine injuries in children, while only forming $16 \%$ of adult cervical spine injuries. ${ }^{1}$ More commonly, these injuries include odontoid fractures, atlanto-occipital dislocation/subluxation and 'hangman' fractures (of the neural arch of C2). ${ }^{1}$ The ligaments most responsible for primary stabilisation of the cranium on the spine are the tectorial membrane (the continuation of the posterior longitudinal ligament up to the basion) and the paired alar ligaments (from the odontoid process to the occipital condyles). ${ }^{4,5}$ Atlanto-occipital dislocation (AOD) occurs more frequently in children than in adults. ${ }^{1,3,6} \mathrm{AOD}$ was considered rare, but new reports claim that it is a major contributor to mortality in paediatric trauma. ${ }^{2,3,6}$ Lower cervical spine distraction is, however, not as frequently described in children under eight years of age. ${ }^{1}$ The mechanism of injury involves combined hyperextension and distraction. ${ }^{3}$
The youngest patients with $\mathrm{AOD}$ are seen following difficult obstetrical deliveries using forceps and after vigorous shaking of neonates in nonaccidental injury. ${ }^{1}$ Children beyond the neonatal period almost invariably sustain $A O D$ as a result of motor vehicle accidents either as passengers or as pedestrians. ${ }^{2,3,4}$ The clinical findings are varied, but AOD usually results in death from brainstem or cord transaction with resultant cardiorespiratory arrest. Eighteen cases have been reported where the patients survived more than 48 hours, as researched and reported by Shamoun et al. ${ }^{3}$ Less severe injuries with instability rather than distraction have also been described with minimal neurological deficit. ${ }^{4,5}$ Lower cervical spine distraction injury can lead to quadriplegia. $2,3,4$ At least 25 reports of AOD have been made, but no significant series of lower cervical spine distraction was found. ${ }^{2,3,4,5,6}$

Methods of imaging include lateral cervical spine radiographs and CT scanning (using the digital 'scanogram' and multiplanar reconstruction), and MRI is used to assess cord damage in survivors. MRI may also provide accurate identification of the cranio-cervical ligaments injured. It may also be useful in differentiating between full and partial ligament injury. Measurements and techniques to aid diagnosis have been controversial. The method of Powers et al calculates a ratio of the distance between the basion and the posterior arch of atlas over the distance between the opisthion and the anterior arch of the atlas. A normal value is considered to be $<7.7$, while any value $>1$ is considered diagnostic of AOD. ${ }^{2,3,4}$ Kaufman et al measure the distance from the 


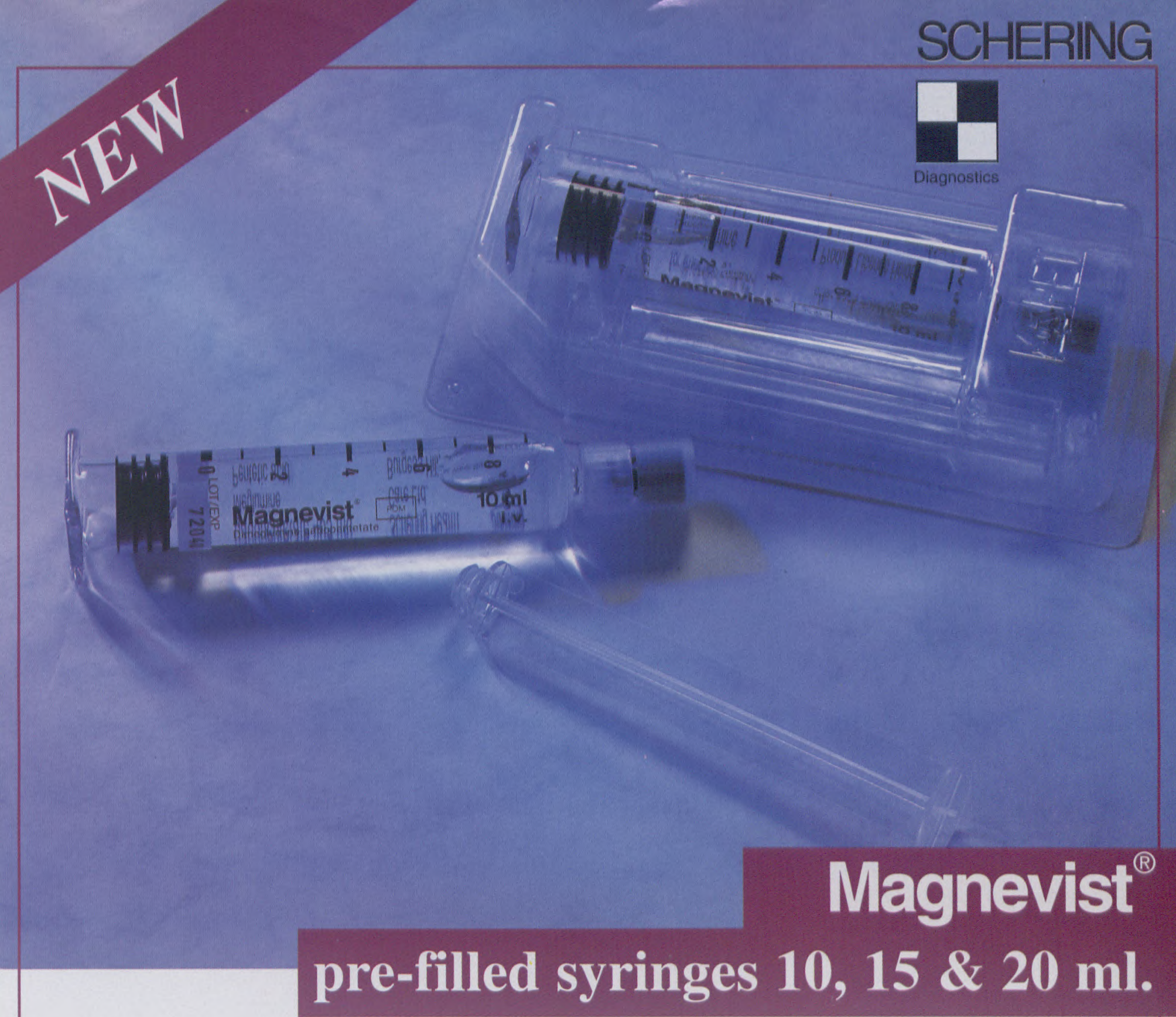

\section{pre-filled syringes $10,15 \& 20 \mathrm{ml}$.}

- CONVENIENCE

Schering understands that timing, accuracy and efficacy are of essence in MRI

- SIMPLICITY

Pre-filled syringes offer fast and easy application

\section{- EASE OF USE}

Injected volume instantly visible through reverse scaling

- Another innovative product in the Schering Diagnostics range.

Schering (Pty) Ltd (Reg. No. : 64/09072/07) 106 Sixteenth Road, Randjespark, Midrand 1685. P.O. Box 5278, Halfway House 1685. UIO 3198 


\section{from page 10}

superior facets of the atlas to the occipital condyles, and consider any value $>5 \mathrm{~mm}$ as abnormal. ${ }^{2}$ The BD distance is that between the basion (B) and the dens (D) and is recorded by Georgopoulos et al to be $5 \mathrm{~mm}$ in a normal adult. ${ }^{4}$ Bulas et al compared the different methods and concluded that the BD distance is the most reliable, with a mean measurement of $8.3 \mathrm{~mm}$ in children. ${ }^{2}$

The post mortem work by Bucholz and Burkhead is misrepresented by both Bulas et al and Shamoun et al. Only three of the 20 children in the Bucholz and Burkhead study had AOD (15\%), and not nine as reported by Bulas et al. ${ }^{2,6}$ Shamoun et al quote that $33 \%$ of the cervical spine injuries in the same series were AODs, but fail to add that this figure included adult patients. ${ }^{3,6}$ However, a five-year review by Shamoun et al reports 10 cases of $\mathrm{AOD}$, representing $18 \%$ of all trauma deaths in their institution. There is also a comment in the same paper that one quarter of patients dying from cervical spine injuries have AOD. ${ }^{3}$

\section{Conclusion}

We present five cases of paediatric cervical spine distraction injury accumulated randomly over a twoyear period. Three of the children had AOD and died within 12 hours of admission to hospital. One of these was a nine-year-old, who should have attained adult characteristics to the cervical spine. The other two cases presented are rare, as they involve distraction occurring at lower cervical spine in children less than four years of age. One of these children died, while the other survives as a quadriplegic. Reports of lower cervical spine distraction in children are scarce, but this case report supports the modern literature, which claims that $\mathrm{AOD}$ is not rare and that it is a significant contributor to paediatric mortality in cervical spine trauma. The cases of lower cervical spine distraction in children younger than eight years are presented as examples of a rarer form of the same process.

\section{References}

1. Sherek HH, Schut L, Lane JM. Fractures and dislocations of the cervical spine in children. Orthop Clin North Am 1976; 9 (3): 593-604.

2. Bulas DI, Fitz CR, Johnson DL. Traumatic atlanto-occipital dislocation in children. Radiology 1993; 188 (1): 155-157.

3. Shamoun JM, Riddick L, Powell RW. Atlanto-occipital subluxation/dislocation: A "survivable" injury in children. Am Surg 1999; 65: 317-320.

4. Georgopoulos G, Pizzutillo PD, Lee MS. Occipito-atlantal instability in children. $J$ Bone and Joint Surg 1987; 69A (3): 429-436.

5. Grabb BC, Frye TA, Hedlund GL, Vaid YN, Grabb PA, Royal SA. MRI diagnosis of suspected atlanto-occipital dissociation in childhood. Pediatr Radiol 1999; 29: 275 281.

6. Bucholz RW, Burkhead WZ. The pathological anatomy of fatal atlantooccipital dislocations. J Bone and Joint Surg 1979; 61 A (2): 248-250. 